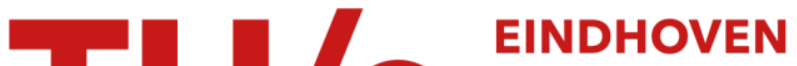 UNIVERSITY OF TECHNOLOGY
}

\section{Exciton enhancement of spin relaxation in diluted magnetic semiconductor quantum wells}

Citation for published version (APA):

Smits, C. J.P., Driel, van, S. C. A., Kampen, van, M., Jonge, de, W. J. M., Koopmans, B., \& Karczewski, G. (2004). Exciton enhancement of spin relaxation in diluted magnetic semiconductor quantum wells. Physical Review B, 70(11), 115307-1/8. [115307]. https://doi.org/10.1103/PhysRevB.70.115307

DOI:

10.1103/PhysRevB.70.115307

Document status and date:

Published: 01/01/2004

\section{Document Version:}

Publisher's PDF, also known as Version of Record (includes final page, issue and volume numbers)

\section{Please check the document version of this publication:}

- A submitted manuscript is the version of the article upon submission and before peer-review. There can be important differences between the submitted version and the official published version of record. People interested in the research are advised to contact the author for the final version of the publication, or visit the $\mathrm{DOI}$ to the publisher's website.

- The final author version and the galley proof are versions of the publication after peer review.

- The final published version features the final layout of the paper including the volume, issue and page numbers.

Link to publication

\section{General rights}

Copyright and moral rights for the publications made accessible in the public portal are retained by the authors and/or other copyright owners and it is a condition of accessing publications that users recognise and abide by the legal requirements associated with these rights.

- Users may download and print one copy of any publication from the public portal for the purpose of private study or research.

- You may not further distribute the material or use it for any profit-making activity or commercial gain

- You may freely distribute the URL identifying the publication in the public portal.

If the publication is distributed under the terms of Article 25fa of the Dutch Copyright Act, indicated by the "Taverne" license above, please follow below link for the End User Agreement:

www.tue.nl/taverne

Take down policy

If you believe that this document breaches copyright please contact us at:

openaccess@tue.nl

providing details and we will investigate your claim. 


\title{
Excitonic enhancement of spin relaxation in diluted magnetic semiconductor quantum wells
}

\author{
C. J. P. Smits,* S. C. A. van Driel, M. van Kampen, W. J. M. de Jonge, and B. Koopmans \\ Department of Applied Physics and center for NanoMaterials (cNM), Eindhoven University of Technology, P.O. Box 513, \\ $5600 \mathrm{MB}$ Eindhoven, The Netherlands \\ G. Karczewski \\ Institute of Physics, Polish Academy of Sciences, Al. Lotników 32/46, 02-668 Warszawa, Poland
}

(Received 14 April 2004; published 14 September 2004)

\begin{abstract}
We performed pump-probe magneto-optical Kerr spectroscopy to explore the role of excitonic effects on the electron and hole spin relaxation in $\mathrm{Cd}_{1-x} \mathrm{Mn}_{x}$ Te-based quantum well structures. Particularly, important information is obtained from detailed interpretation of the temperature and wavelength dependence of the observed transient magneto-optics in specially engineered structures. In manganese-rich wells the electron spin relaxation is found to be strongly enhanced in the exciton due to the increased mass of the bound electron-hole pair, in agreement with earlier predictions. An even stronger enhancement of the relaxation time is found for the hole spin, which is explained by the additional mixing of heavy-hole and light-hole states in the bound state. In structures where $\mathrm{Mn}$ is confined to the barrier region, a different behavior is observed, indicative for a competition of various contributions.
\end{abstract}

DOI: 10.1103/PhysRevB.70.115307

PACS number(s): 78.67.De, 71.35.Gg, 78.47.+p, 76.30.Pk

\section{INTRODUCTION}

Injection and manipulation of spin polarized carriers into semiconductor structures, so-called spintronics, has become a hot issue in both magnetism and semiconductor communities. ${ }^{1}$ Novel device options are being envisioned once sufficient control over the electron spin in hybrid structures is achieved. Diluted and ferromagnetic semiconductors can be considered ideal components of such devices, combining magnetic and semiconducting properties. Much interest has been devoted to achieve spin injection using ferromagnetic semiconductors such as GaMnAs, ${ }^{2}$ as well as spin filtering using diluted magnetic semiconductors (e.g., CdMnTe). ${ }^{3}$

While the spin dynamics in III-V nonmagnetic semiconductors, particularly GaAs and GaAs/AlGaAs quantum well structures, has been well studied over the past decade, ${ }^{4-6}$ relatively little attention has been devoted to II-VI diluted magnetic semiconductor (DMS) structures, such as the aforementioned CdMnTe. ${ }^{7}$ In particular, a complete insight in the temperature dependence of the spin relaxation, as well as a detailed experimental attempt to compare spin dynamics of free carriers as opposed to excitons is lacking. In this paper we report on such an investigation, using a time-resolved pump-probe magneto-optical technique.

Several processes contribute to the electron spin relaxation in nonmagnetic III/V and II/VI semiconductors and quantum well structures. ${ }^{8}$ In semiconductors that lack a center of inversion symmetry, the electron spin relaxation is often dominated by the D'Yakonov-Perel (DP) mechanism, ${ }^{9,10}$ an effect associated with the spin splitting of the conduction band. For centrosymmetric materials, or if momentum scattering is significant, spin-orbit induced Elliott-Yaffet (EY) relaxation ${ }^{11}$ may take over the dominant role. Finally, for $p$-type materials, the high hole density may promote efficient electron-hole scattering, denoted after Bir, Aharonov, and Pikus (BAP). ${ }^{12}$ Excitonic binding of electrons and holes at low temperatures may be another reason for an increased BAP contribution.

Dynamics of the hole spin is quite contrasting to that of the electron spin. Due to the finite orbital momentum carried by the holes, spin-orbit scattering is a potentially efficient scattering mechanism for the hole spin leading to high relaxation rates. The splitting of the valence band for heavy holes (HH's) and light holes (LH's) in quantum well structures, however, tends to strongly quench this effect, leading to an interesting balance of competing mechanisms at low temperatures. ${ }^{13}$

In DMS materials an additional efficient mechanism is exchange scattering of electrons with the local magnetic moments of the magnetic ions, $s-d$ and $p-d$ scattering. More specifically for II-VI DMS, Akimoto et al. ${ }^{14}$ performed experiments on $\mathrm{CdTe} / \mathrm{Cd}_{1-x} \mathrm{Mn}_{x} \mathrm{Te}$ quantum wells, with magnetic manganese ions only in the barrier. A correlation between electron spin relaxation rate and overlap of the wave function with Mn-rich barriers was concluded from the correlation between spin relaxation and confinement energy. An even more direct relation between electron spin relaxation and $\mathrm{Mn}$ concentration came from experiments by Koopmans et al. ${ }^{15}$ on series of $\mathrm{Cd}_{1-x} \mathrm{Mn}_{x} \mathrm{Te} / \mathrm{Cd}_{1-x-y} \mathrm{Mn}_{x+y} \mathrm{Te}$ quantum wells, in which the confinement energy was kept constant while varying the Mn concentration in the well. In an alternative approach the role of manganese in the spin relaxation process can be inferred from measuring the spin dynamics of the local manganese atoms, instead of the conduction electrons. ${ }^{16}$ As to the hole spin, Akimoto ${ }^{14}$ found no dependence of its relaxation on the well width, explained by the competition of the consequences of wave function penetration into the barrier and increasing HH-LH splitting at increasing confinement. Finally, Camilleri et al. ${ }^{17}$ reported on electron and hole spin relaxation in both $n$-type and $p$-type CdMnTe quantum wells with nonmagnetic barriers. The electron spin-relaxation rate was compared with predictions by Bastard et al., ${ }^{18}$ who derived a simple expression for the 
relaxation in infinitely deep quantum wells due to $s$ - $d$ scattering. A satisfactory agreement was found at low temperatures $(\sim 5 \mathrm{~K})$, provided that the exciton mass was used rather than the effective band mass of the free electron. Apart from this indirect relationship, we are not aware of any direct evidence of exciton effects to the spin relaxation in II/VI DMS structures. In this context we would like to mention that distinct dynamics has been observed for excitons and trions in nonmagnetic II-VI semiconductors. ${ }^{19}$

In Sec. II of this paper we describe the structures studied with both magnetic and nonmagnetic quantum wells, and briefly review our experimental approach denoted as timeresolved magnetization modulation spectroscopy (TiMMS). The results are reported in Sec. III. We address the basic characterization (covering spectroscopic features and interferometric artifacts, separation of electron and hole signals, magnetic field and fluence dependence) in Sec. III A. A detailed analysis of the electron and hole spin relaxation as a function of temperature is given in Sec. III B, followed by a presentation of experimental data evidencing a strong photon energy dependence of the observed spin relaxation in Sec. III C. Our experimental findings are discussed in Sec. IV, with a strong emphasis on the role of excitonic effects. Finally, conclusions and an outlook are provided in the last section.

\section{EXPERIMENTAL}

Various $\mathrm{Cd}_{1-x} \mathrm{Mn}_{x}$ Te quantum well structures were investigated, in which the magnetic manganese impurities were either incorporated inside the quantum well or in the barrier material. The local band gaps at the quantum wells were adjusted further by adding magnesium. Typical band gaps are $1.6-1.7 \mathrm{eV}$ in the quantum well, tuned to fit within our laser energy range. Quantum well widths range from 6-10 nm. All samples were grown by molecular beam epitaxy (MBE) along the [001] crystal axis on $\mathrm{GaAs}(001)$ substrates. CdTe and $\mathrm{Cd}_{0.75} \mathrm{Mg}_{0.25} \mathrm{Te}$ were used as buffer layers, and the quantum wells were capped with CdMgTe.

The paper will focus mainly on two exemplary samples serving as representatives for all samples measured, for convenience to be denoted sample A and B. Sample A consists of a single 8 -nm-thick $\mathrm{Cd}_{0.995} \mathrm{Mn}_{0.005}$ Te quantum well grown on a $\mathrm{GaAs}(0.5 \mathrm{~mm}) / \mathrm{Cd}_{0.75} \mathrm{Mg}_{0.25} \mathrm{Te}(4.2 \mu \mathrm{m})$ substrate with a $0.3-\mu \mathrm{m} \mathrm{Cd}{ }_{0.85} \mathrm{Mg}_{0.15} \mathrm{Te}$ buffer layer in between. The quantum well is capped with a $0.1-\mu$ m-thick $\mathrm{Cd}_{0.85} \mathrm{Mg}_{0.15} \mathrm{Te}$ layer. Sample B contains ten 8-nm-thick $\mathrm{Cd}_{0.96} \mathrm{Mn}_{0.04} \mathrm{Te}$ quantum wells separated by 25 -nm-thick $\mathrm{Cd}_{0.85} \mathrm{Mg}_{0.15}$ Te barriers. It was grown on the same substrate using the same buffer and capping layers as sample A. Photoluminescence measurements at $5 \mathrm{~K}$ show peaks at $1.637 \mathrm{eV}$ for sample A and $1.709 \mathrm{eV}$ for sample B.

The magnetization dynamics of these samples were measured using TiMMS, time-resolved magnetization modulation spectroscopy, an optical pump-probe technique that is described in detail by Koopmans et al. ${ }^{20}$ In short, a pulse from a tunable Ti:sapphire laser, is split in two separate pulses with intensity ratio of around 5:1. The high intensity pump pulse is used to spin selectively excite electrons in the quantum wells. The time-delayed, weaker probe pulse is then used to measure the magneto-optical Kerr rotation. Alternatively, the Kerr ellipticity is resolved if a quarter-wave plate is put in the probe beam, just after the sample. ${ }^{15}$

Spin-selective excitation is achieved by guiding the pump pulses through a photoelastic modulator (PEM), introducing a circular polarization with a handedness oscillating at $50 \mathrm{kHz}$. The probe pulses are modulated by placing a mechanical chopper in the probe beam, operating at a frequency of typically $60 \mathrm{~Hz}$. The measured Kerr signal is then led through a series of two lock-in amplifiers, of which the first one is set at the modulation frequency of the PEM and the second one at the frequency of the chopper in the probe beam. Because of this double modulation any possible signal due to pump light reaching the detector is completely suppressed. ${ }^{20}$

The samples were mounted in an optical flow cryostat, allowing measurements in the temperature range of 5-300 K. In order to increase the spectral resolution in the TiMMS experiment, in some of our experiments a monochromator was placed in the probe beam, between the sample and the photodiode detector. This way the spectral resolution is increased from typically $10 \mathrm{~nm}$ to around $1 \mathrm{~nm}$, allowing to resolve spectral features such as the exciton from the free electrons. Although the enhanced spectral selectivity is accompanied by an equivalent loss in time resolution (from typically $100 \mathrm{fs}$ to $1 \mathrm{ps}$ ), this is not a serious limitation for the ps spin dynamics we concentrate on in this paper.

\section{RESULTS}

\section{A. Basic characterization}

TiMMS spectra obtained for sample A (a single quantum well on a transparent buffer layer) at $5 \mathrm{~K}$ and at a fixed time delay (5 ps) are displayed in Fig. 1(a). This specific sample configuration may be considered ideal as to study spin dynamics in the quantum well; the optical band gap of the barrier layer is chosen higher than the photon energy so that absorption is restricted to the quantum well region. Ellipticity and rotation are explicitly measured separately. ${ }^{15}$ The respective spectra are found to be nicely related via a KramersKronig transformation. A resonant structure near the band gap $E_{0}$ is observed, in agreement with the photoluminescence spectrum. As expected, the resonant structure shifts monotonously as a function of temperature, as shown in Fig. 1(b) for the peak position of the rotation spectrum.

While the spectrum is well measurable, one might hope to increase the magneto-optical signal by performing reflection experiments on a multiquantum well structure such as sample B. Figure 1(c) displays a typical magneto-optical spectrum showing an oscillatory structure. One might be inclined to assign certain features in the spectrum to the successive peaks. However, spectra measured at different temperatures indicate that the positions of the peaks do not follow the temperature dependence of the band gap. This can be seen in Fig. 1(d), which shows the peak positions as a function of temperature. Two parallel effects are visible: first a gradual temperature dependence of the envelope of the oscillatory magneto-optical signal that follows the shift in 

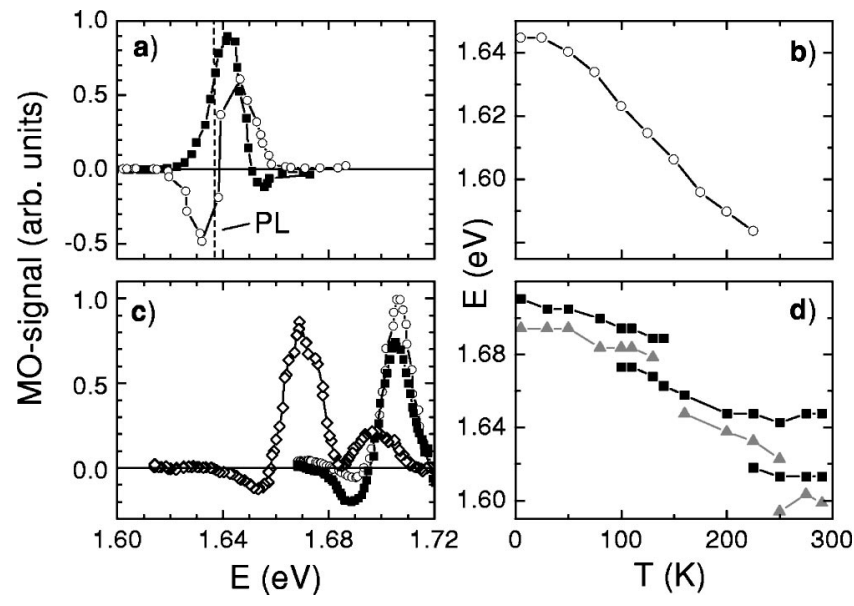

FIG. 1. Spectrum of the magneto-optical signal and its temperature dependence at zero field: Panel (a) shows both rotation (open circles) and ellipticity (closed squares) for the single quantum well containing $0.5 \% \mathrm{Mn}$ (sample A) as measured at $T=5 \mathrm{~K}$, and in panel (b) the temperature dependence of the maximum in the rotation spectrum is shown. Panel (c) shows the magneto-optical spectrum for a multiple quantum well (sample B) with $4 \% \mathrm{Mn}$ in the well at a temperature of 5 (open circles), 80 (closed squares), and $160 \mathrm{~K}$ (open diamonds). In panel (d) the temperature dependence of the extrema in the magneto-optical spectrum of the multiple quantum well is shown, with black squares denoting maxima and gray triangles denoting minima.

band gap, and secondly a shift of the peaks within the envelope. The latter effect is well explained by interferometric effects. Interference causes an additional dependence of the spectrum on the refractive index in the material, which is in its turn dependent on the temperature.

More detailed modeling showed that in fact the magnetooptical signal is made up out of a nonmagnetic contribution, dominated by reflection at the outer surface, and a magnetic signal that is coherently being built up by two transmissions (up and down) through the ten quantum wells, and internally reflecting at the buffer/substrate interface. Although the reflection at the latter interface is relatively small, this is compensated by the efficient and cumulative effect of the Faraday rotation in this quasitransmission configuration. Explicit modeling ${ }^{21}$ properly resolves the thickness of the overlayer $(4.9 \mu \mathrm{m})$ from the fringe period. One may nevertheless use this configuration for a wavelength-dependent measurement of spin relaxation times, though utmost care has to be taken in identifying features in the magneto-optical spectrum.

While part of the complication discussed above may thought to be solved by performing experiments on non transparant buffer layers, such as pure CdTe, this results in optical excitation of $e-h$ pairs in the buffer layer. Consequently, additional TiMMS signals not related to the one from the quantum well under consideration may complicate the analysis, especially for structures with a band gap relatively close to that of CdTe. A separation of electron-spin signals is often still possible due to the difference in $g$ factor. However, hole spin identification gets more troublesome. Therefore, for these structures our analysis will be limited to electron spin relaxation.
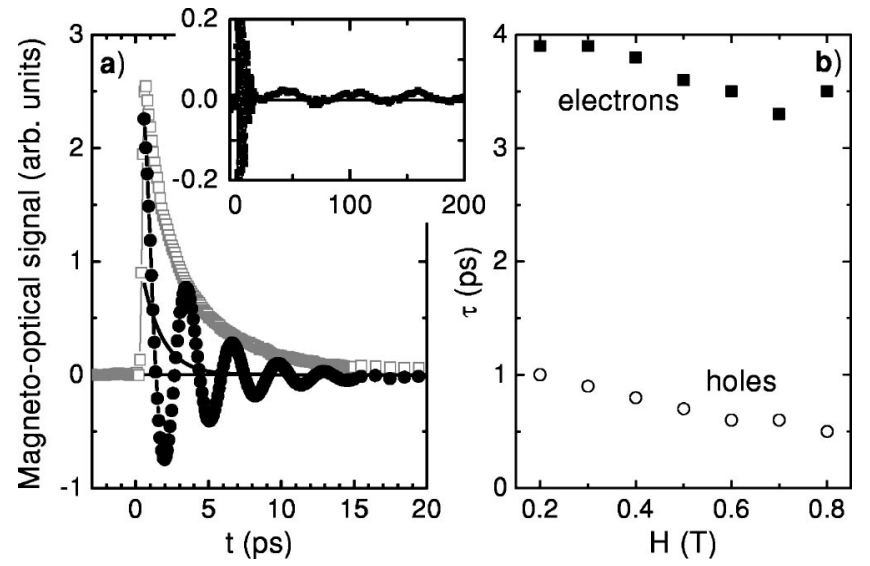

FIG. 2. (a) Magneto-optical signal as a function of the time after the arrival of the pump pulse for sample B (the multiple quantum well containing $4 \% \mathrm{Mn}$ ) at $5 \mathrm{~K}$. Open gray squares represent a zero-field measurement, whereas closed circles correspond to measurements under an applied magnetic field of $0.65 \mathrm{~T}$. The solid line is the nonoscillating part of a fit of the in-field measurement with one exponentially decaying component for the holes and one oscillating component with an exponentially decaying amplitude for the electrons, Eq. (1). (b) Electron and hole spin relaxation times as a function of the applied transverse magnetic field for the same sample, also at $5 \mathrm{~K}$.

The procedure of electron and hole-spin signal separation in the Voigt configuration, measuring the transverse spin lifetime, is illustrated in Fig. 2(a) for sample B. In the presence of an in-plane magnetic field, in this case of $0.65 \mathrm{~T}$, the optically injected electrons will perform a precessive motion with a characteristic Larmor frequency directly related to the electron $g$ factor. However, in a quantum well, the hole spins are frozen by the confining potential, unless the magnetic field is high enough to overcome the heavy hole-light hole splitting. Thus the total signal, representative for the spin component perpendicular to the film plane, is a superposition of an exponentially decaying oscillatory (electron) and nonoscillatory (hole) signal. The induced magneto-optical rotation as a function of time $\Theta(t)$ at an arbitrary excitation energy can thus be written as

$$
\Theta(t)=A_{e} \sin \left(\omega_{L} t\right) \exp \left(-t / \tau_{e}\right)+A_{h} \exp \left(-t / \tau_{h}\right)
$$

where $\tau_{e}$ and $\tau_{h}$ denote the electron and hole spin relaxation times, respectively, and $\omega_{L}$ the electron Larmor frequency. The fit to the data resolves equal amplitudes for electron $\left(A_{e}\right)$ and hole $\left(A_{h}\right)$ within satisfactory precision. In passing we want to note that at longer delay times a weaker oscillatory signal with $g=2$ remains [inset, Fig. 2(a)] as a consequence of the local manganese $d$ moments that have been polarized by the optically injected carriers. ${ }^{7,17}$

Although a magnetic field can thus be used to resolve electron and hole spin relaxation, one has to carefully evaluate whether the magnetic field itself does not affect the relaxation process. Therefore, careful experiments as a function of magnetic field have been performed. As a first test, Fig. 2(a) also includes a result at zero magnetic field. The resulting spin signal agrees relatively well with the envelope 


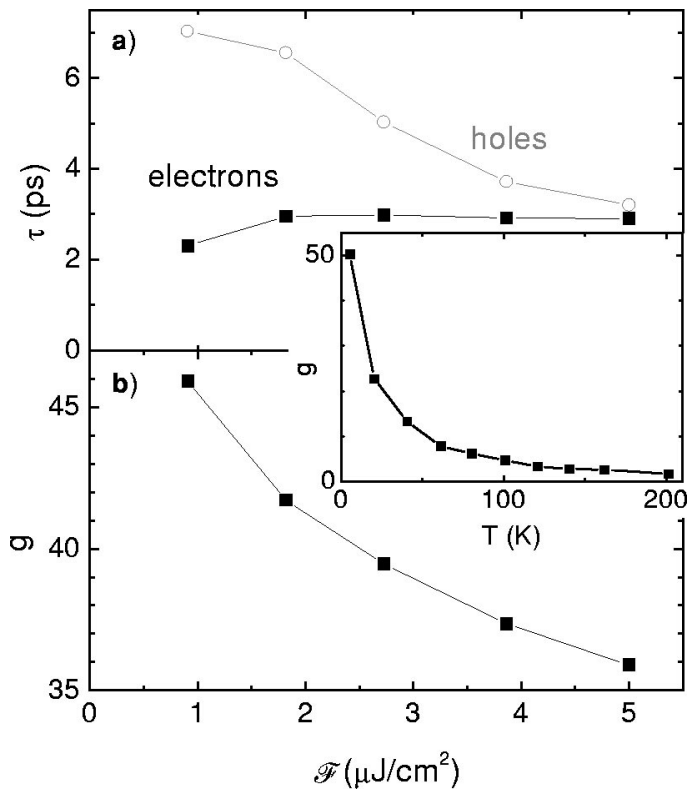

FIG. 3. (a) Electron and hole spin relaxation times as a function of laser fluence used for the spin-selective excitation for the multiple $4 \% \mathrm{Mn}$ quantum well at $5 \mathrm{~K}$ (sample B). (b) The fluence dependence of the electron $g$ factor for the same sample, again at $5 \mathrm{~K}$. The inset shows for a comparison the temperature dependence of the $g$ factor. All measurements were performed under the application of an in-plane magnetic field of $0.8 \mathrm{~T}$.

of the in-field data. More detailed data are represented in Fig. 2(b), showing the electron and hole spin relaxation time versus field. It is found that the electron spin relaxation is constant within $10 \%$ for fields up to $1 \mathrm{~T}$. Consequently, the separation method provides a relatively field-independent value of the electron spin decay time $\tau_{e}$, and dephasing effects are found to be insignificant. In contrast, the hole spin relaxation time is reduced by a factor of 2 at $0.8 \mathrm{~T}$, in agreement with earlier work. ${ }^{7}$ This effect can be assigned to the field induced mixing of heavy hole and light hole states. Care has thus to be taken in discussing hole spin relaxation.

Finally, it has to be verified whether the excitation densities used in our experiments $\left(5 \mu \mathrm{J} / \mathrm{cm}^{2}\right)$ affect the spin relaxation. In general, for the electron signal, the spin dynamics was found to be relatively independent of laser fluence, as illustrated in Fig. 3(a). However, in particular at low temperatures we did find a strong dependence of the hole spin relaxation on laser fluence, also illustrated in Fig. 3(a). In these data obtained at $5 \mathrm{~K}$ for sample B (4\% Mn), a reduction of $\tau_{h}$ by a factor of 2 at our maximum fluence was observed. This effect could be traced back to a minor laser heating in combination with the extreme temperature dependence of $\tau_{h}$ that is observed for some of our samples in this temperature regime. The effect of laser heating can be well estimated from a measurement of the electron $g$ factor, which shows a strong temperature dependence for DMS materials. In the inset of Fig. 3(b) the electron $g$ factor of sample B is plotted versus temperature, as determined from measurements at a low laser fluence in an applied in-plane magnetic field. The $g$ factor drops monotonously from 50 at $5 \mathrm{~K}$ to 2 at 200 K. Figure 3(b) shows the fluence dependence of the $g$

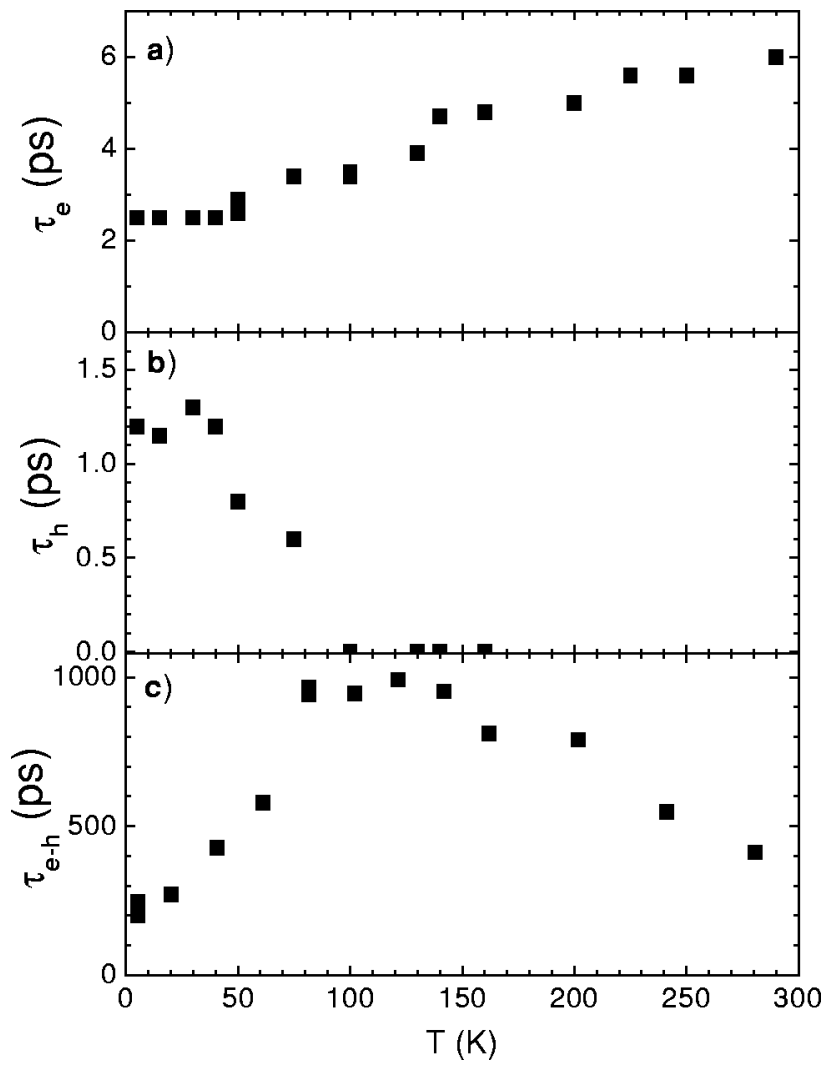

FIG. 4. Electron (a) and hole (b) spin relaxation time, measured at $0.8 \mathrm{~T}$, as well as the electron-hole recombination time at zero field (c) as a function of temperature for the $4 \%$ Mn multiple quantum well (sample B).

factor at $T=5 \mathrm{~K}$. The observed decrease by $20 \%$ can be related to a laser-induced temperature rise of approximately $5 \mathrm{~K}$ at the highest fluence. As will be discussed in Sec. III B this has a minor effect on the electron spin relaxation time, but can have a large impact on the hole spin relaxation time.

\section{B. Temperature dependence}

Important information on the mechanisms relevant for the electron and hole spin relaxation can be obtained by detailed studies of its temperature dependence. An exemplary case is reproduced in Fig. 4. Figures 4(a) and 4(b) display the electron and hole spin relaxation time, respectively, for sample B as a function of temperature, as derived from fitting the TiMMS time traces at an external field of $0.80 \mathrm{~T}$. At each temperature, the laser energy was tuned to the first peak in the TiMMS ellipticity spectrum. The hole spin relaxation proceeds within a few picoseconds at $5 \mathrm{~K}$, while it virtually vanishes at temperatures above $100 \mathrm{~K}$. The electron spin relaxation time shows a somewhat counterintuitive behavior: it increases monotonously by a factor of three from $2.5 \mathrm{ps}$ to $8 \mathrm{ps}$ while raising the temperature from 5 to $200 \mathrm{~K}$. It was carefully checked that this was the genuine behavior for a near band gap excitation and detection, and it was reproduced for other samples as discussed below.

In order to correctly interpret the spin relaxation rates obtained it should be verified whether electron-hole recom- 


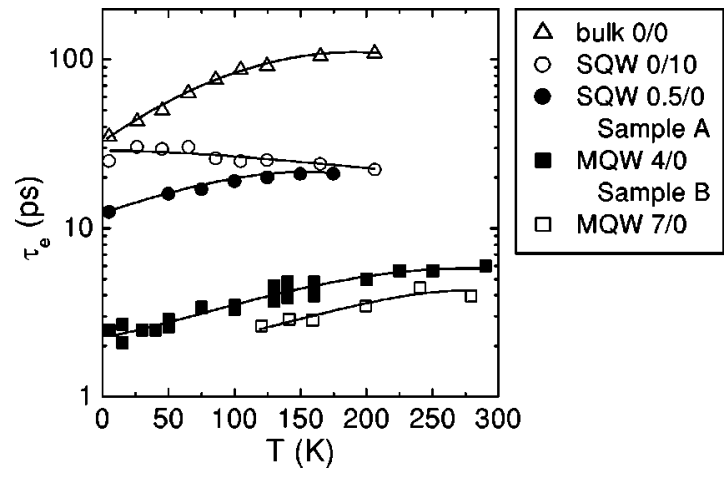

FIG. 5. Electron spin relaxation time as a function of temperature for several quantum well structures. Samples are denoted by two numbers of which the first denotes the manganese content inside the well, whereas the second indicates the amount of manganese in the barrier. Furthermore, A and B indicate samples A and B, respectively. The temperature behavior of the spin relaxation in bulk CdTe is also given for comparison. The lines are only meant as guides to the eye.

bination influences the observed dynamics. As an example, the $e-h$ recombination times extracted from transient reflection measurements are depicted in Fig. 4(c). From 5 to $100 \mathrm{~K}$ a slowdown of the recombination is observed, related to the tendency of carriers to distribute over a wider region in $\vec{k}$ space, which hinders efficient recombination. At higher temperatures a reversed trend is indicative for defectrelated recombination. Independent of the underlying mechanisms, we found for all our samples that the recombination time is sufficiently large compared to the electron and hole spin relaxation time to be able to consider the density of both carrier types constant during the spin relaxation process.

An overview of the temperature dependence of the electron and hole spin relaxation times for various magnetic wells, barriers, and bulk CdTe is given in Fig. 5. An increase of the electron spin relaxation with temperature is observed for all the magnetic wells. In contrast, the sample with the manganese-rich barrier shows an "ordinary" decrease of spin relaxation with temperature, i.e., a faster relaxation at higher temperatures. Finally, the CdTe bulk sample has again an inverse behavior, though at much slower rates. Overall, a spectacular increase of the spin relaxation rate with Mn content of the structure is witnessed.

The hole spin relaxation is shown in Fig. 4(b) for sample B. Because of difficulties in discriminating the hole spin dynamics from the total signal due to the absence of hole spin precession, as mentioned before, only a limited amount of data has been included. Overall, a strong reduction of spin relaxation time with temperature is found, reducing to the sub-hundreds of femtoseconds for temperatures over $100 \mathrm{~K}$. As mentioned before, around $5 \mathrm{~K}$ a strong dependence on laser fluence has been found, related to minor laser heating. It should be noted that the temperatures in the diagram can be a slight underestimate of the actual temperature, since laser heating (maximum $5 \mathrm{~K}$ at a base temperature of $5 \mathrm{~K}$ ) has not been accounted for.

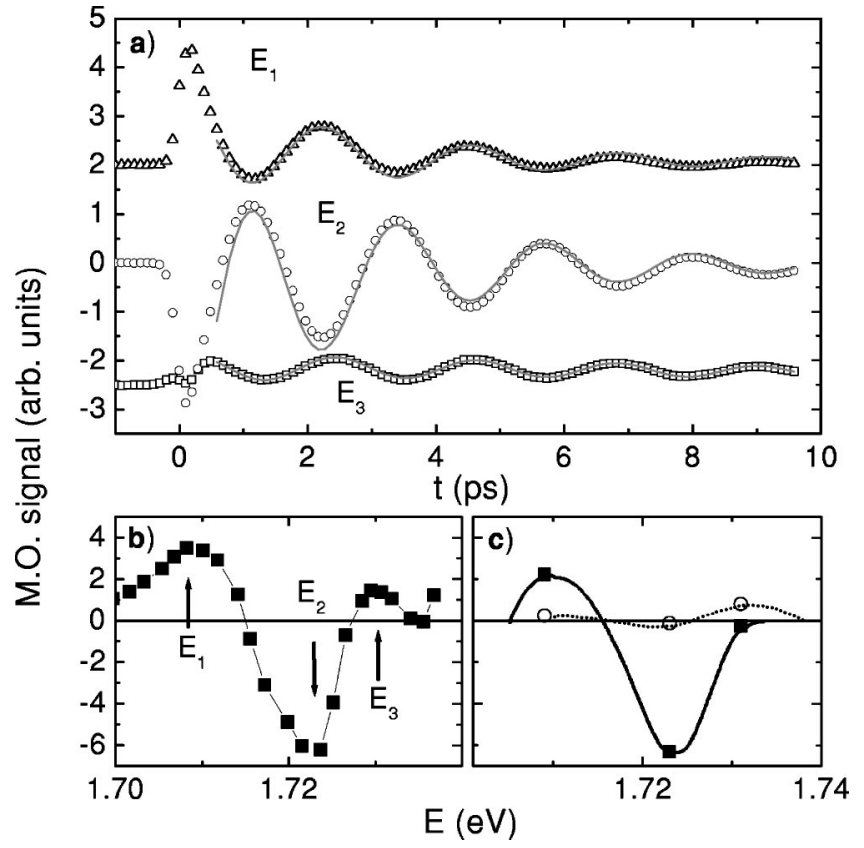

FIG. 6. (a) The magneto-optical signal as a function of the time after excitation at excitation energies of 1.709 (triangles), 1.723 (circles), and $1.731 \mathrm{eV}$ (squares) for a $4 \% \mathrm{Mn}$ multiple quantum well at an applied magnetic field of $0.8 \mathrm{~T}$ at $5 \mathrm{~K}$. The drawn lines are fits with a superposition of two sine functions with damping amplitude and two simple exponentials superimposed. The offsets were added in order to spatially separate the different graphs. (b) The spectral dependence of the magneto-optical signal at a constant delay time of $3 \mathrm{ps}$ in the absence of a magnetic field. The explicit time dependencies were measured at the extrema of this spectrum. (c) The amplitudes of the different constituents to the magnetooptical signal as obtained from the fits in panel (a). Open circles indicate free electrons, whereas full squares denote excitons. The lines are guides to the eye.

\section{Photon energy dependence}

Bastard et al. ${ }^{18}$ have suggested that the spin relaxation times in diluted magnetic semiconductors should be significantly higher for free electrons and holes than for excitons. Therefore we may assign, as a working hypothesis, the "inverse" temperature dependence of the magnetic barrier samples to dissociation of excitons at elevated temperatures. The exciton binding energy in bulk CdTe is approximately $10 \mathrm{meV}$, and will be slightly higher in the quantum wells under consideration here. This translates to a characteristic temperature of approximately $120 \mathrm{~K}$, in good agreement with the observed temperature profiles. If this hypothesis were to be taken seriously, one should in principle be able to find a different spin relaxation time at different excitation energies. Excitation well enough above the exciton transition energy will predominantly lead to free carriers. If the exciton formation time is not negligible with respect to the spin relaxation time, our degenerate TiMMS experiment might provide different spin relaxation times depending on excitation energy.

In Fig. 6(a), TiMMS transients measured at three characteristic energies in the spectrum [see Fig. 6(b)] are displayed. 
TABLE I. Spin relaxation times (at $5 \mathrm{~K}$ ) for electrons and holes in the quantum wells of sample B as obtained from the fits in Fig. 6.

\begin{tabular}{cc}
\hline \hline & Spin relaxation $(\mathrm{ps})$ \\
\hline$\tau_{e, \text { exc }}$ & 3.1 \\
$\tau_{e, \text { free }}$ & 7.7 \\
$\tau_{h, \text { exc }}$ & 1.2 \\
$\tau_{h, \text { free }}$ & 27.2 \\
\hline \hline
\end{tabular}

Even at a first glance it is visible that the transient TiMMS signals depend strongly on excitation energy. In general, a faster relaxation is found when exciting right at the band edge as compared to excitation a higher energies. It turns out to be possible to fit the three sets of curves with two sets of time constants $\left(\tau_{e}, \tau_{h}\right)$, as introduced in Eq. (1). In view of a possible interpretation in terms of excitons and free carriers, we label the fitted time constants as $\tau_{e, \text { exc }}, \tau_{e \text {,free, }} \tau_{h, \text { exc }}$, and $\tau_{h, \text { free, }}$ respectively. As a constraint we required the corresponding initial amplitudes to be equal for electrons and holes, $A_{e, \mathrm{exc}}=A_{h, \mathrm{exc}}$ and $A_{e, \mathrm{free}}=A_{h, \mathrm{free}}$, because the same number of electrons and holes is excited. The photon energy dependence of the amplitudes $A_{e, \text { exc }}(\hbar \omega)$ (squares) and $A_{e, \text { free }}(\hbar \omega)$ (circles) is depicted in Fig. 6(c). The fitted relaxation times are given in Table I.

As one of the detailed outcomes to be discussed in the forthcoming section, the "high-energy" electron spin relaxation (tentatively assigned "free" and labeled $\tau_{e, \text { free }}$ ) agrees fairly well with the high temperature $(200 \mathrm{~K})$ value obtained right at the band gap (see Fig. 4).

Similar energy dependencies have been measured at different temperatures and for different samples. However, when increasing temperatures above $100-150 \mathrm{~K}$, no significant energy dependence has been observed. This would be in agreement with dissociation of excitons at elevated temperatures. Though, it should be mentioned that the limited wavelength range of our laser did not allow for a fully systematic study in this regime.

\section{DISCUSSION}

Bastard and Ferreira ${ }^{18}$ predicted an enhancement of the $s-d$ exchange scattering in the excitonic state already more than ten years ago, based on calculations of the electron spin relaxation rate by Bastard and Chang. ${ }^{22}$ For an infinitely deep quantum well of width $w$ with an effective manganese content of $x_{\text {eff }}$, they predicted for the electron spin relaxation time

$$
\frac{1}{\tau_{e}}=\frac{35}{16} \frac{m_{\mathrm{eff}} J^{2}}{\hbar^{3}} \frac{4 x_{\mathrm{eff}}}{w},
$$

where $m_{\text {eff }}$ is the effective mass of the electron or, alternatively, of the exciton under consideration. Furthermore the exchange constant for $s-d$ interaction between electrons and manganese moments $J$ equals $0.22 \mathrm{eV} \times a_{0}^{3} / 4$, with $a_{0}$ being $6.487 \AA$, and $\hbar$ is Dirac's constant. Various approximations of $x_{\text {eff }}$ to account for antiferromagnetic interactions between

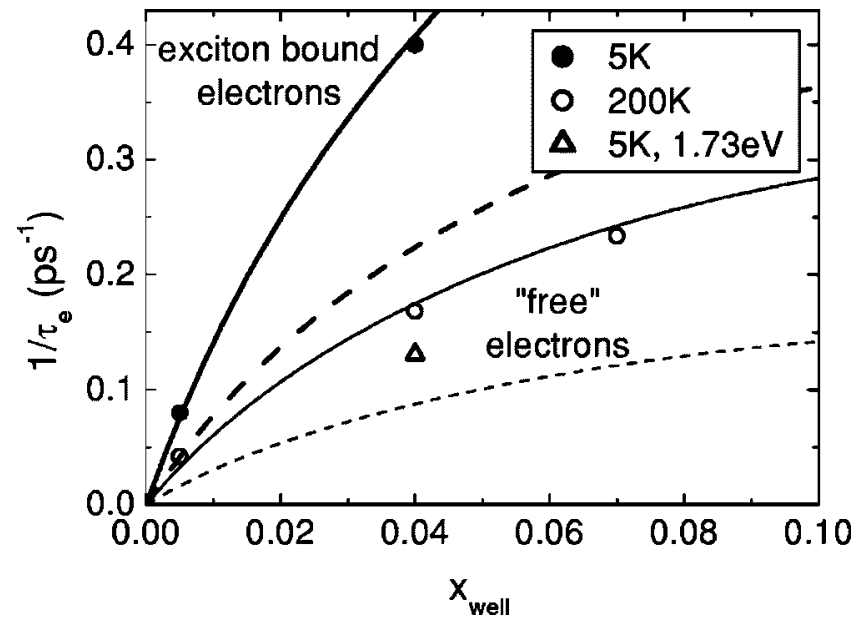

FIG. 7. Electron spin relaxation rates for 8-nm-thick quantum wells containing various concentrations of manganese both at low temperatures $(5 \mathrm{~K})$ and at room temperature. The lines represent the calculations by Bastard and Chang (Ref. 22), corrected for antiferromagnetic interactions between manganese ions, following the expression proposed by Gaj et al. (Ref. 23). Dashed lines indicate the parameter-free results, whereas solid lines represent the results after application of a global scaling parameter of 1.8. For comparison also a data point at $5 \mathrm{~K}$ using a higher excitation energy is given.

neighboring manganese spins can be found in literature. Here we use $x_{\text {eff }}(x)=x\left(0.01+0.73 e^{-6.2 x}+0.26 e^{-43.3 x}\right)$ as proposed by Gaj et al. ${ }^{23}$ The circles in Fig. 7 show the measured electron spin relaxation rates for samples with a varying amount of manganese in the quantum wells, after excitation close to the band gap. The scattering rate rises with manganese content, an indication of relaxation by $s-d$ exchange scattering. Both measurements at 5 and at $200 \mathrm{~K}$ are presented, the latter temperature being well above the exciton dissociation temperature of approximately $120 \mathrm{~K}$. The dashed lines represent the parameter free model by Bastard and Chang ${ }^{22}$ [Eq. (2)] for the distinct dynamics of exciton bound and free carriers by using literature values for the effective mass $\left[m_{\text {exc }}\right.$ $=0.246 m_{e}$ and $m_{\text {free }}=0.096 m_{e}$ (Ref. 24)]. One might expect that such a simple model can only yield a qualitative, orderof-magnitude estimate of the dynamics. However, just by introducing a single overall scaling factor of 1.8 , reasonably close to unity, the solid lines are obtained that overlap all data points to a surprisingly high accuracy. The lowtemperature data fall onto the curve for exciton bound electrons, whereas those at $200 \mathrm{~K}$ match the curve for free electrons.

Moreover, in Fig. 7 a measurement of $\tau_{e}$ at low temperature $(5 \mathrm{~K})$, but at a higher excitation energy $E_{3}$, see Fig. 6(b), is added (indicated by a triangle). The spin relaxation time is found to be longer than for excitation at the resonance wavelength and resembles values measured at high temperatures, where the exciton state is no longer stable. This again suggests that the formation of excitons reduces the electron spin relaxation time.

In distinguishing between the different relaxation mechanisms, the temperature dependence of the spin relaxation is of great value. The temperature dependence of the electron spin relaxation was shown in Fig. 5. First of all, for the 
manganese rich quantum wells, where the strongest spin relaxation mechanism is the $s$ - $d$ scattering on the manganese impurities, we observe an increase of the spin relaxation time with increasing temperature. The temperature dependence is in qualitative agreement with the formation and dissociation of excitons, as already discussed in the previous paragraph.

Having assigned $s$ - $d$ scattering as the main contribution for the manganese rich wells, we next turn our attention to the Mn-rich barriers. These samples display a strikingly different temperature dependence. We believe that the net behavior is a rather complex mixture of different effects. First of all, the total spin relaxation is significantly slower, allowing for other spin relaxation channels to be of importance. Moreover, the formation of excitons has a secondary effect for the structures, not discussed so far. In the excitonic state, the additional confining Coulomb interaction binding the electron and hole, causes a reduction of the penetration of the electron and hole wave functions into the barrier. Thus the overlap of the wave functions with the manganese rich barriers is reduced. Since the latter overlap is thought to be the main contribution to the spin relaxation, ${ }^{15,18,22}$ exciton dissociation at elevated temperatures should speed up the spin relaxation, as is indeed observed for these structures. More detailed studies are still needed for a firmer assignment, however.

The bulk CdTe again displays the inverse temperature dependence of $\tau_{e}$. Clearly, $s-d$ exchange has no influence in the nonmagnetic compound, and a comparison with spin relaxation in GaAs is more appropriate. In low or moderately doped GaAs, spin relaxation is dominated by the DP mechanism, which scales inversely proportional to the momentum relaxation ${ }^{9} \tau_{e} \propto \tau_{p}^{-1}$. Thus, as the temperature is increased, the DP mechanism becomes less effective due to an increased momentum relaxation, leading to longer spin relaxation times. In passing, we note that at even higher temperatures, not covered by our experiments, a crossover to the EY spin relaxation mechanism is anticipated, leading to a faster spin relaxation again. A clue for the decrease of $\tau_{e}$ at low temperatures (below $50 \mathrm{~K}$ ) may be obtained from speculations of exciton-enhanced $e-h$ scattering (BAP mechanism) in GaAs (Ref. 25) and ZnCdSe. ${ }^{26}$ Compared to the GaAs case such an effect should be even stronger in the more polar $\mathrm{CdTe}$, and range up to higher temperatures.

Although our discussion mainly focusses on the electron spin, our limited data set on the hole spin does allow for some interesting speculations. It is well known that the hole spin relaxation in bulk nonmagnetic materials is extremely fast, which is caused by an EY-like process, in which due to spin-orbit coupling and the heavy hole-light hole degeneracy orbital momentum can act as an efficient source/sink of spin momentum. Alternatively, in the quantum well the HH-LH splitting hinders this relaxation channel. Though, at finite temperatures states at larger $\vec{k}$ values are being populated. Since the HH-LH anticrossing is located at relatively low $k$ values, this rapidly leads to an efficient mixing of $\mathrm{HH}$ and LH states and recovery of the fast hole spin relaxation. ${ }^{13}$

The $p$ - $d$ exchange interaction behaves in a similar way, being formally forbidden in quantum wells at zero temperature, but rapidly increasing in importance at elevated temperature. Thus, both mechanisms could satisfactory explain the fast decrease of $\tau_{h}$ with temperature [Fig. 4(b)]. More spectacular, however, is the extreme reduction of the hole spin relaxation rate when going from the bound exciton state to the free holes, as evidenced by the spectroscopic TiMMS results (Table I). Part of this effect could be due to the different exciton mass as compared to the free heavy hole mass. However, this difference is smaller than that for the electron mass. Therefore, we conjecture that it is the large spread in $\vec{k}$ space needed to form a localized exciton that gives rise to a more efficient coupling of heavy hole and light hole wave functions, and thus a faster hole spin relaxation. In this tentative interpretation, the confinement-induced HH-LH splitting, which tends to increase $\tau_{h}$, competes with excitonconfinement enhanced HH-LH coupling. As to a final assessment of this scenario, as well as the relative importance of $s-d$ exchange and EY scattering, more systematic studies are still needed.

\section{CONCLUSION}

In conclusion, the role of excitonic effects to the electron and hole spin relaxation in $\mathrm{Cd}_{1-x} \mathrm{Mn}_{x}$ Te-based quantum well structures was investigated by means of time-resolved magnetization modulation spectroscopy (TiMMS). Exciton states were distinguished from those of free electron and holes by their different position in the magneto-optical spectrum, and by the observation that they are only present at low temperatures. The electron spin relaxation in manganese-rich wells turned out to be much faster in the exciton due to the increased mass of the bound electron-hole pair, which confirms earlier predictions. The hole spin relaxation rate was enhanced even more, which can be explained by the additional mixing of heavy-hole and light-hole states in the bound state. Overall, we find a satisfactory agreement of the observed spin relaxation time with the parameter-free model of Bastard. ${ }^{18,22}$ In structures where the manganese was confined to the barrier region, the electron spin relaxation increased with temperature due to the higher penetration of the electron wave function in the barrier. The excitons could contribute to this trend by increasing the confinement of the spins inside the quantum wells.

\section{ACKNOWLEDGMENTS}

This work is part of the research programme of the "Stichting voor Fundamenteel Onderzoek der Materie (FOM)" which is financially supported by the "Nederlandse Organisatie voor Wetenschappelijk Onderzoek (NWO)." 
*Electronic address: C.J.P.Smits@tue.nl

${ }^{1}$ S. A. Wolf, D. D. Awschalom, R. A. Buhrman, J. M. Daughton, S. von Molnár, M. L. Roukes, A. Y. Chtchelkanova, and D. M. Treger, Science 294, 1488 (2001).

${ }^{2}$ Y. Ohno, D. K. Young, B. Beschoten, F. Matsukura, H. Ohno, and D. D. Awschalom, Nature (London) 402, 790 (1999).

${ }^{3}$ R. Fiederling, M. Keim, G. Reuscher, W. Ossau, G. Schmidt, A. Waag, and L. W. Molenkamp, Nature (London) 402, 787 (1999).

${ }^{4}$ S. Bar-Ad and I. Bar-Joseph, Phys. Rev. Lett. 68, 349 (1992).

${ }^{5}$ A. Tackeuchi, Y. Nishikawa, and O. Wada, Appl. Phys. Lett. 68, 797 (1996).

${ }^{6}$ R. S. Britton, T. Grevatt, A. Malinowski, R. T. Harley, P. Perozzo, A. R. Cameron, and A. Miller, Appl. Phys. Lett. 73, 2140 (1998).

${ }^{7}$ S. A. Crooker, D. D. Awschalom, J. J. Baumberg, F. Flack, and N. Samarth, Phys. Rev. B 56, 7574 (1997).

${ }^{8}$ J. Fabian and S. Das Sarma, J. Vac. Sci. Technol. B 17, 1708 (1999).

${ }^{9}$ M. I. D’yakonov and V. I. Perel', Zh. Eksp. Teor. Fiz. 60, 1954 (1971) [Sov. Phys. JETP 33, 1053 (1971)]; Fiz. Tverd. Tela (Leningrad) 13, 3581 (1971) [Sov. Phys. Solid State 13, 3023 (1972)].

${ }^{10}$ M. I. D’yakonov and V. Yu. Kachorovskii, Fiz. Tekh. Poluprovodn. (S.-Peterburg) 20, 178 (1986) [Sov. Phys. Semicond. 20, 110 (1986)].

${ }^{11}$ R. J. Elliott, Phys. Rev. 96, 266 (1954).

${ }^{12}$ G. L. Bir, A. G. Aronov, G. E. Pikus, Zh. Eksp. Teor. Fiz. 69, 1382 (1975) [Sov. Phys. JETP 42, 705 (1976)].

${ }^{13}$ R. Ferreira and G. Bastard, Phys. Rev. B 43, 9687 (1991).

${ }^{14}$ R. Akimoto, K. Ando, F. Sasaki, S. Kobayashi, and T. Tani, Phys. Rev. B 56, 9726 (1997).

${ }^{15}$ B. Koopmans, M. van Kampen, and W. J. M. de Jonge, Phys. Status Solidi B 215, 217 (1999).

${ }^{16}$ A. V. Akimov, A. V. Scherbakov, D. R. Yakovlev, W. Ossau, L.
W. Molenkamp, T. Wojtowicz, J. Kossut, S. Tatarenko, and J. Cibert, Physica B 316-317, 41 (2002).

${ }^{17}$ C. Camilleri, F. Teppe, D. Scalbert, Y. G. Semenov, M. Nawrocki, M. Dyakonov, J. Cibert, S. Tatarenko, and T. Wojtowicz, Phys. Rev. B 64, 085331 (2001).

${ }^{18}$ G. Bastard and R. Ferreira, Surf. Sci. 267, 335 (1992).

${ }^{19}$ J. Tribollet, F. Bernardot, M. Menant, G. Karczewski, C. Testelin, and M. Chamarro, Phys. Rev. B 68, 235316 (2003).

${ }^{20}$ B. Koopmans, J. E. M. Haverkort, W. J. M. de Jonge, and G. Karczewski, J. Appl. Phys. 85, 6763 (1999).

${ }^{21}$ For a maximum in the interference pattern the optical path inside the material should be an integer times the light wavelength. Maxima thus exist for $d=m\left(\lambda_{m} / 2 n\right)$, where $d$ is the layer thickness, $\lambda_{m}$ the light wavelength, $n$ the refractive index of the material at wavelength $\lambda$, and $m$ an integer. From the difference in wavelength between two adjacent maxima $\Delta \lambda / \lambda=(2 n d / \lambda$ $+1)^{-1}$, it follows that the distance between two maxima, $16.7 \mathrm{~nm}$, leads to a layer thickness of $4.9 \mu \mathrm{m}$ for a refractive index of 3.5. When the wavelength dependence of the refractive index is taken into account, a refractive index of 3 would account for the distance between the maxima in the interference pattern, a reasonable value for a layer mainly consisting of $\mathrm{Cd}_{0.75} \mathrm{Mg}_{0.25} \mathrm{Te}$.

${ }^{22}$ G. Bastard and L. L. Chang, Phys. Rev. B 41, 7899 (1990).

${ }^{23}$ J. A. Gaj, W. Grieshaber, C. Bodin-Deshayes, J. Cibert, G. Feuillet, Y. Merle-d'Aubigne, and A. Wasiela, Phys. Rev. B 50, 5512 (1994).

${ }^{24}$ Numerical Data and Functional Relationships in Science and Technology, Landolt-Börnstein, Vol. III-17b (Springer-Verlag, Berlin, 1982).

${ }^{25}$ A. Malinowski, R. S. Britton, T. Grevatt, R. T. Harley, D. A. Ritchie, and M. Y. Simmons, Phys. Rev. B 62, 13034 (2000).

${ }^{26}$ J. M. Kikkawa, I. P. Smorchkova, N. Samarth, and D. D. Awschalom, Science 277, 1284 (1997). 